\title{
Compress sensing algorithm for estimation of signals in sensor networks
}

 \\ José Antonio Marmolejo-Saucedo ${ }^{3}$ (i)
}

(C) Springer Science+Business Media, LLC, part of Springer Nature 2019

\begin{abstract}
In this research, we present a data recovery scheme for wireless sensor networks. In some sensor networks, each node must be able to recover the complete information of the network, which leads to the problem of the high cost of energy in communication and storage of information. We proposed a modified gossip algorithm for acquire distributed measurements and communicate the information across all nodes of the network using compressive sampling and Gossip algorithms to compact the data to be stored and transmitted through a network. The experimental results on synthetic data show that the proposed method reconstruct better the signal and in less iterations than with a similar method using a thresholding algorithm.
\end{abstract}

Keywords Sensor networks · Compressive sampling · Gossip algorithms

\section{Introduction}

Sensor networks, both wired and wireless, have found a wide variety of applications, which has caused their growth, and with this, a greater amount of information to propagate [23]. Thus, there exists a constantly search for optimization in both speed and in the amount of data to be transmitted. In a network with thousands of nodes, for unify their information it would be necessary at least $n$ transmissions, which $n$ is the number of nodes, in practice this could be very slow or not at all useful, also this can carry out other problems such as: complications to detect possible failures, maintenance, or even to detect possible attacks on the network [12]. To solve this problems, an alternative is the use of compressed sensing

Electronic supplementary material The online version of this article (https://doi.org/10.1007/s11276-019-02031-5) contains supplementary material, which is available to authorized users.

Oliverio Cruz-Mejía

ocruzm@uaemex.mx

1 Universidad Autónoma de Ciudad Juárez, Ciudad Juárez, Mexico

2 Universidad Autónoma del Estado de México, Toluca, Mexico

3 Facultad de Ingeniería, Universidad Panamericana, Augusto Rodin 498, 03920 Ciudad de México, Mexico
(CS) algorithms [4, 10], which seek to reconstruct a signal starting from a much smaller amount of data, this is possible by expressing the signal in sparse domain (Fourier, Wavelet, etc.), where most of its elements are null, this could be beneficial in both speed, amount of data to be transmitted, power savings and facilitate the analysis of information.

The use of optimization and CS has been used in a distributed setting in several works. In [21] it is presented a distributed projected consensus algorithm were nodes combine their local average with projection on their individual constraint sets. In [19] are proposed several strategies based on distributed iterated hard thresholding algorithm over a network that employ diffusion mechanisms, the developed algorithms have low complexity in terms of communication in the network. In the work of [11] is proposed the use of approximated message passing algorithm to reduce the amount of data transmitted in the sensor network, this algorithm try to reduce the communication cost, while maintaining the same recovery solution as the centralized scheme. However, their algorithm relies on the knowledge of the data of each sensor, thus the performance decays when the number of sensor increases. In [25] a distributed greedy algorithm for sparse learning is proposed, the algorithm is not based in a consensus scheme but instead it is designed to achieve efficiency through cooperation and information exchange. In [6] a framework for distributed minimization of non-convex 
functions is proposed, such framework is based on differences of gradients using successive convex approximations and the use of consensus to distribute the computation among network nodes, each node solves a local convex approximation problem following by local averaging operations. The work [20] introduced an algorithm based on distributed inexact gradient and gradient tracking techniques, and doubly stochastic mixing matrices, at each step of the algorithm all nodes iterate to a global and consensual minimum.

All the aforementioned algorithms, make use of a type of consensus optimization paradigm, in this research we explore gossip schemes for efficient implementation of CS algorithms in the distributed setting to achieve consensus within the nodes in the network, in order to reduce the computational complexity and minimize the number of active nodes at each time step.

Here, we proposed a distributed CS scheme for application in sensor networks to solve the problem of signal estimation, and where each node has only available a few measurements through linear incoherent sampling. Our proposed scheme is based on gossip algorithms and approximated message passing, this allows a rapid convergence over a sensor network and reduce the computational complexity as compared with the work of [11]. To the best of our knowledge, the proposed scheme is the first to combine gossip algorithms with the approximated message passing in a distributed setting. Our main contribution is a novel algorithm that combines approximated message passing and gossip methods. The proposed algorithm is robust to diverse topologies, in the sense that it can work even if each node has only one neighbor, also, in the proposed scheme each step is easy to compute. This study is limited to sensor networks with at least one communication link per node.

The rest of the paper is organized as follows. In the Sect. 2, the CS and gossip algorithms are reviewed. In the Sect. 3 the proposed algorithm is presented. The Sect. 4 details the experiments, and results are presented. Finally, the conclusions are provided in the Sect. 5.

\section{Theory}

In this work we represents the network of $N$ nodes as a connected graph $G=(V, E)$, where $V=\{1,2, \ldots, N\}$ is a set of nodes, and $E \subset V \times V$, represents the set of communication links between two nodes (edges). Also, we denote the data at each node $v \in V$ at a time $t$ as $x_{v}(t)$.

\subsection{Gossip algorithms}

Gossip algorithms for distributed systems were introduced for reliable transfer data in communication systems. In a network using these algorithms, information is exchanged asynchronously and no specialized routing algorithm is necessary $[1,15]$. If all nodes in a network have access to a subset of the data, then, under the Gossip scheme, information is exchanged iteratively between a subset of nodes at a time, until all information is propagated [5]. At each step a node share information with a neighbor to compute a local update. Here we are interested in pairwise randomized gossiping [3]. One classic application example is the so called average consensus, which consists of distributed averaging in the network given by $G$ where each node $v \in V$ has an initial measurement $x_{v}(0)$ and it is required that the whole network knows and estimates the average of all the measurements of the nodes of $G$ [7]. Then, at each iteration of the algorithm the following steps are done:

1. A pair of nodes $(v, w) \in E$ are selected randomly.

2. The selected nodes exchange their current estimates, $x_{v}(t), x_{w}(t)$.

3. Each node updates their estimates as

$$
x_{v}(t+1)=x_{w}(t+1)=\left(x_{v}(t)+x_{w}(t)\right) / 2 .
$$

Convergence of the pairwise gossip algorithm to the true average is guarantee if the nodes keep gossiping each other for enough time [7].

\subsection{Compressed sensing}

The theory of CS has been integrated in many areas of image and signal processing and communications. There are CS applications in MRI [16], signal compression [17], radar [14], cognitive radio [24], among others.

Given a compressible signal, CS techniques are able to reconstruct that signal using only few linear measurements $[2,4]$. Considering a signal $x \in \mathbb{R}^{n}$ that is $\mathrm{k}$-sparse, that is where only $k$ of $n$ coefficients are nonzero and unknown, then using CS theory it is possible to reconstruct $x$ from a measurement vector $y \in \mathbb{R}^{m}$, where $m<<n$, obtained through linear sampling using a measurement matrix $A, C S$ theory establishes conditions for selecting a suitable $A$ that guarantees an optimal reconstruction. A common choice that works with high probability is to select $A$ as a matrix of random numbers, then $x$ can be recovered exactly or approximately from the measurements $y$ by solving

$\hat{x}=\underset{x}{\operatorname{argmin}}\|x\|_{0} \quad$ such that $\quad\|A x-y\| \leq \epsilon$,

were $\epsilon>0$ accounts for error on the measurements $y$. It is a convex optimization problem known as basis pursuit $[2,13,18]$. There exist several techniques that can be used to solve (2). Here we are interested in solving (2) under a distributed setting. 


\section{Methods}

In this paper we consider the problem of estimate a signal $x \in \mathbb{R}^{n}$ employing a sensor network $G$, where each node $v$ of $G$ has available measurements $y_{v} \in \mathbb{R}^{m}$ given by

$y_{v}=A_{v} x+e_{v}$,

here $m<<n, A_{v} \in \mathbb{R}^{m \times n}, e_{v}$ is a Gaussian noise, and $x$ is assumed to be $k$-sparse. Thus, one way to estimate the signal $x$ at each node is solving the following optimization problem

$\hat{x}=\underset{x}{\operatorname{argmin}} \sum_{v \in V}\left\|y_{v}-A_{v} x\right\|_{2}^{2} \quad$ such that $\|x\|_{0}<k$

This can be solved using CS theory in a distributed setting, the nodes in the network could exchange information, helping to attain an estimate of the signal in a faster and with better precision as compared to non-distributed setting [19].

To solve the optimization problem using a collaborative scheme, Gossip Pairwise algorithms are combined with threshold reconstruction methods, this leads to obtain an approximation of the signal to be recovered in each iteration of the gossip algorithm.

There exists several algorithms for distributed reconstruction, in which, each node obtains an approximation of the original signal that is updated iteratively according to the information provided by the neighbors, one of them is the iterative hard thresholding (IHT) with gossip [22]. Here we propose an algorithm based on the optimization scheme called Approximate Message Passing Algorithm (AMP) [8, 9], which is an improvement of the methods based on a thresholding schemes, it adds a term based on the theory of belief propagation in graphical models. The updates of the AMP algorithm are as follows

$x(t+1)=\sigma_{t}\left(A^{T} z(t)+x(t)\right)$,

where $\sigma_{t}$ is a thresholding function such as soft thresholding and $z(t)$ is the residue between the encoded information and the estimates
$z(t)=y-A x(t)+m(t)$,

where $m(t)$ is a term that acts as a momentum and it is one of the key modifications over IHT technique, this term is given by

$m(t)=\frac{1}{\delta} z(t-1)\left\langle\sigma_{t}^{\prime}\left(A^{T} z(t-1)+x(t-1)\right)\right\rangle$,

with $\sigma_{t}^{\prime}$ being the derivative of the threshold function and

$\langle f\rangle=\sum_{i=1}^{n} \frac{f(i)}{n}$

denotes the sampling mean.

Here we use AMP in conjunction with Gossip to solve the aforementioned problem in a sensor network. Thus, given a pair of nodes $v$ and $w$ selected by the gossip algorithm, we calculate the updates for each of the gossiping nodes as

$x_{v}(t+1)=\sigma_{k}\left[\frac{x_{v}(t)+x_{w}(t)}{2}+\tau A_{v}^{T} z_{v}(t)\right]$,

and

$x_{w}(t+1)=\sigma_{k}\left[\frac{x_{v}(t)+x_{w}(t)}{2}+\tau A_{w}^{T} z_{w}(t)\right]$

where we expect that the inclusion of the terms $z_{v}(t)$ and $z_{w}(t)$ will help to achieve a rapid convergence, these terms are computed as follows

$$
\begin{aligned}
z_{v}(t)= & y_{v}-A_{v} \frac{x_{v}(t)+x_{w}(t)}{2} \\
& +\frac{1}{\delta} z_{v}(t-1)\left\langle\sigma_{t}^{\prime}\left(A^{T} z_{v}(t-1)+x_{v}(t-1)\right)\right\rangle, \\
z_{w}(t)= & y_{w}-A_{w} \frac{x_{v}(t)+x_{w}(t)}{2} \\
& +\frac{1}{\delta} z_{w}(t-1)\left\langle\sigma_{t}^{\prime}\left(A^{T} z_{w}(t-1)+x_{w}(t-1)\right)\right\rangle .
\end{aligned}
$$

\begin{tabular}{|c|}
\hline Proposed algorithm \\
\hline 1 Initialize: $x_{v}=0$ for all $v \in V$, set $\tau>0, \mathrm{~m}(0)=0$ \\
\hline 2 for $\mathrm{t}=0,1, \ldots$, stop iter do \\
\hline 3 Select randomly a communication link $E=(u, w)$ \\
\hline $4 z_{v}(t)=y_{v}-A_{v} \frac{x_{v}(t)+x_{w}(t)}{2}+\frac{1}{\delta} z_{v}(t-1)\left\langle\sigma_{t}^{\prime}\left(A^{T} z_{v}(t-1)+x_{v}(t-1)\right)\right\rangle$ \\
\hline $5 \quad x_{v}(t+1)=\sigma_{k}\left[\frac{x_{v}(t)+x_{w}(t)}{2}+\tau A_{v}^{T} z_{v}(t)\right]$ \\
\hline $6 \quad z_{w}(t)=y_{w}-A_{w} \frac{x_{v}(t)+x_{w}(t)}{2}+\frac{1}{\delta} z_{w}(t-1)\left\langle\sigma_{t}^{\prime}\left(A^{T} z_{w}(t-1)+x_{w}(t-1)\right)\right\rangle$ \\
\hline $7 \quad x_{w}(t+1)=\sigma_{k}\left[\frac{x_{v}(t)+x_{w}(t)}{2}+\tau A_{w}^{T} z_{w}(t)\right]$ \\
\hline $8 x_{h}(t+1)=x_{h}(t)$ for any $h \neq v, w$ \\
\hline 9 end \\
\hline
\end{tabular}

In the next section we demonstrate the effectiveness of the proposed method and offer comparisons with Gossip-IHT of [22]. The proposed algorithm is shown below. 


\section{Results}

In this section we present the different experiments carried out during the investigation, as well as the analysis and evaluation of the results obtained. We compare gossip IHT [22] with our proposed method, gossip AMP. We simulated a sensor network with 100 nodes, and we select the communication range of each sensor such that every node has at least one neighbor and there are no nodes without communicating. In Fig. 1 it is shown a realization of a simulated network.

For the experiments, we also simulated a signal with values in the range of $[-1,1]$ and then we use a k-sparse version of it. Additionally, a measurement matrix A was created inline for each node, by using a predefined seed for generating random numbers, this has the advantage of not being expensive in storage. As a measure of error we used the mean squared error.

In the first experiment we generated a 100 samples signal with a sparsity of $k=15$. We use this data to obtain values for the parameters of each algorithm. In the Gossip IHT algorithm the parameter $\tau$ was modified between $[0,1]$, until finding a small recovery error in all the nodes, in this case the mean square error. In Fig. 2, the decrease in the error is shown for several iterations of the gossip IHT algorithm with different values of $\tau$. We show the two curves for which the reconstruction gives the less error, which correspond to $\tau=0.006$ and $\tau=0.007$, the minimum error is attained at iteration 1200 were can be observed that the most suitable value for $\tau$ is 0.007 with an error of 0.000233 .

In the evaluation of the proposed Gossip AMP algorithm we worked with the parameters $\tau$ and $\delta$, which were modified in the intervals $[0,1]$ and $[1,5]$ respectively. In Fig. 2, it is shown the two curves for which the reconstruction gives the less error, that correspond to the combination of parameters: $\tau=0.01$ and $\delta=1$; and $\tau=0.007$

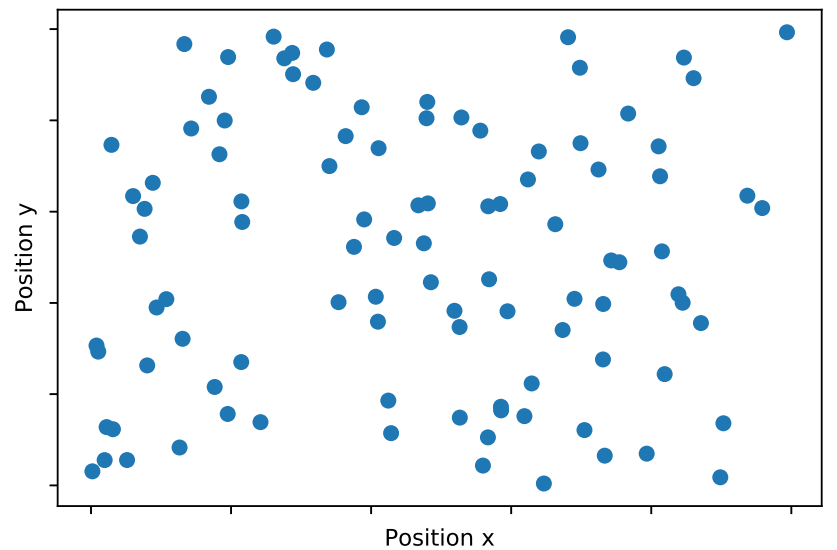

Fig. 1 A realization of a sensor network with 100 nodes

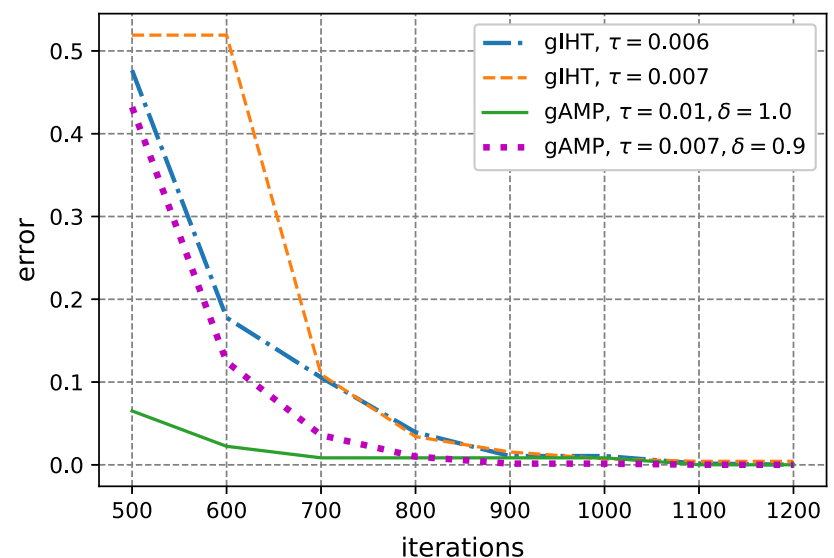

Fig. 2 The graph shows the reconstruction errors of the algorithms for each iteration of the signal. We show the best curves for the gossip IHT (gIHT) and the proposed algorithm, gossip AMP (gAMP)

and $\delta=0.9$. The minimum error at 1200 iterations was achieved with $\tau=0.007$ and $\delta=0.9$ with an error of 0.0000181 .

Individual progress of the estimates during the iterations of the Gossip IHT and AMP algorithms can be visualized in Fig. 3, it can be noted that in the first iterations the gossip IHT algorithm has large oscillations in comparison with the proposed algorithm. In addition, some values never stop oscillating in the IHT algorithm, for example the point near to the value 0.6 , while in the proposed algorithm all the test points converge.

Once the values of the parameters in each algorithm are established, we test again on a new data realization on a new network (100 random nodes). A maximum error was obtained every 100 iterations starting from the 500 until arriving to 1200 , with the purpose of visualizing the progress of the estimations of each algorithm, this is shown in Fig. 4 where it can be observed that Gossip AMP decreases the error faster than Gossip IHT.

To visualize the reconstruction of all the nodes, the signal of each node was overlapped in the same graph for each algorithm, this is shown in Fig. 5.

We also explore the results without knowing the sparsity of the signal before hand. For this end, we generated data to obtain a 100 samples signal in the range of $[0,1]$, next we obtained a sparse version of it by using the Eq. (13)

$\sigma(x ; \theta)=\left\{\begin{array}{cc}(x-\theta) & \text { if } x>\theta \\ 0 & -\theta \leq x \leq \theta \\ (x+\theta) & \text { if } x<-\theta\end{array}\right.$

were we used a value of $\theta=0.001$ to perform the experiments. The parameters of each algorithm were varied within intervals that were determined empirically and that had greater possibility of containing the optimal values. 

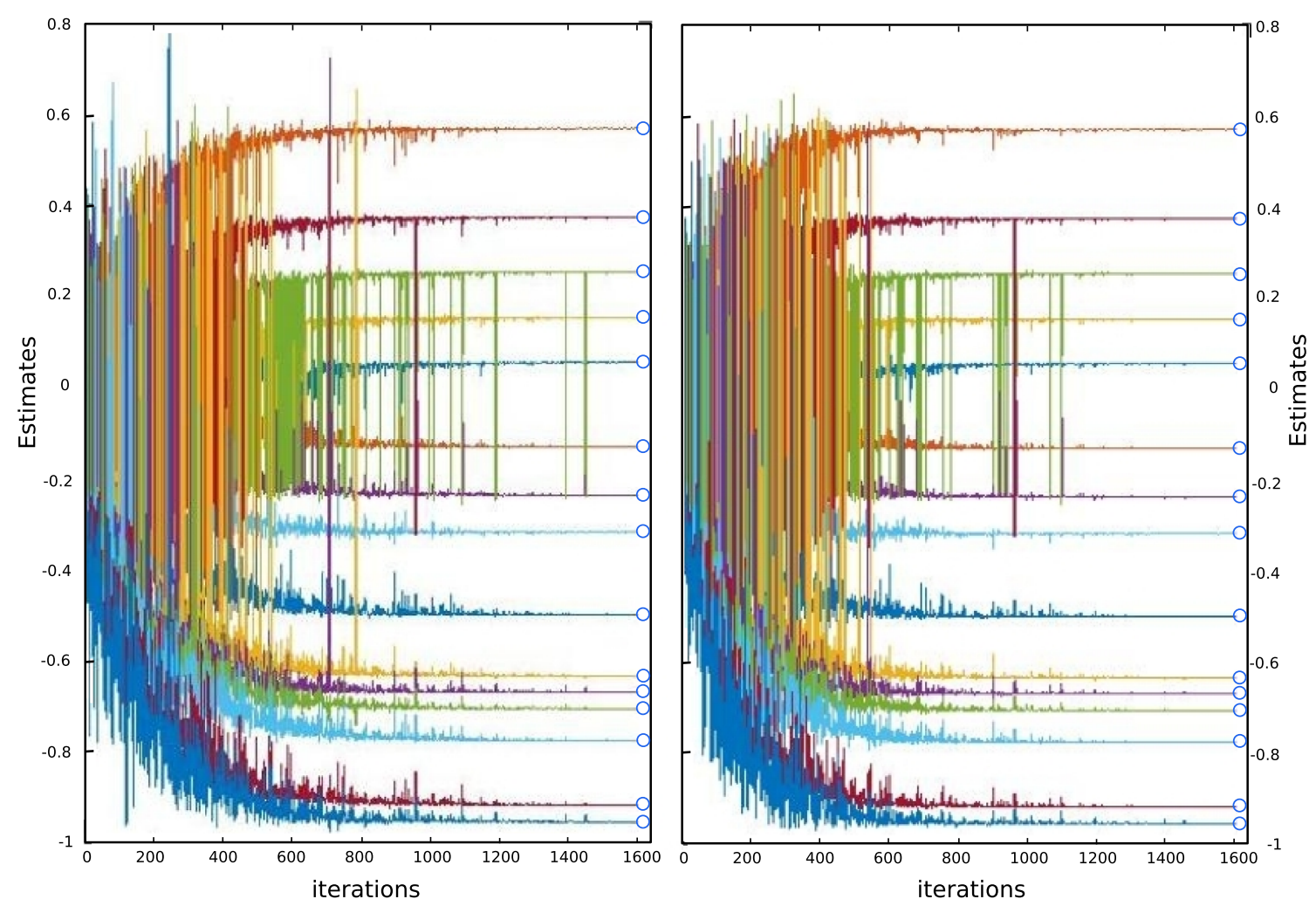

Fig. 3 The graphs show the convergence of the algorithms for 15 points of the signal, whose true value are denoted by the circles. The left graph shows the gossip IHT algorithm, while the left graph shows the proposed algorithm

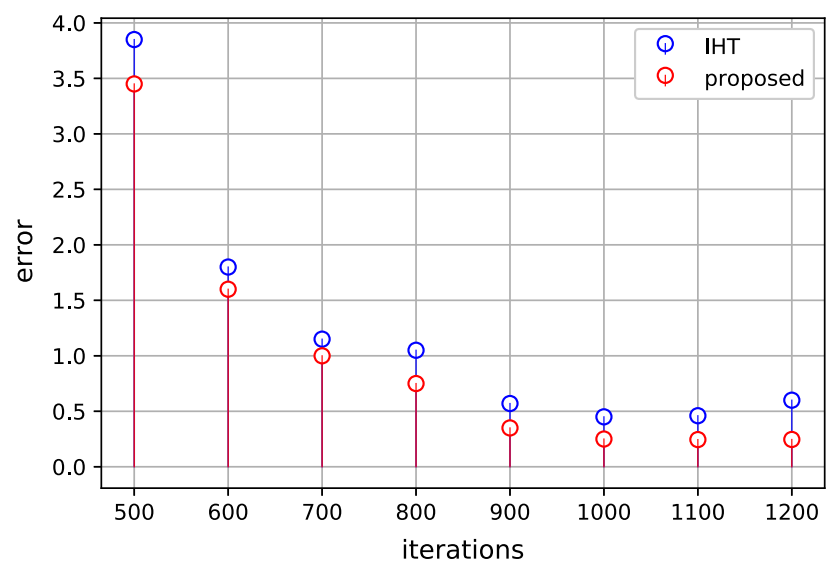

Fig. 4 Progress through iterations of the Gossip AMP algorithm, $\tau=$ 0.006 and $\delta=0.007$

We evaluate the Gossip IHT algorithm for different values of the parameter $\tau$ in a range of $[0,1]$ and for several iterations. In Fig. 6 we can see the decrease of the error in IHT as $\tau$ as varying. We only show the two curves with less error for a given iteration. The best result is found with the value of $\tau=0.005$, with an error of 0.149 . On the other hand, we evaluate the proposed algorithm varying the parameters $\tau$ and $\delta$, which were modified in the intervals $[0,1]$ and $[1,5]$ respectively. Figure 6 shows the two curves with less error. Here the best value is given by an error of 0.02 , using $\tau=0.007$ and $\delta=0.9$.

Also, from Fig. 6 we can see that for a given iteration, the reconstruction error is less by using the proposed algorithm. At iteration 500 the error is twice between the best combination of parameters for the proposed algorithm and the second best combination of parameter for the gossip IHT. We also make comparisons of total time to complete an iteration, for this we used an Intel(R) Core(TM) i7-6500U CPU at $2.50 \mathrm{GHz}$. The time measured per iteration where $0.001103 \mathrm{~s}$ for the gossip IHT and $0.002152 \mathrm{~s}$ for the proposed method, which is almost 1 ms bigger. Although it could affect the performance on a particular real time application, However, it is negligible for non-real time applications.

In Table 1, are shown some advantages and disadvantages of the proposed method.

\section{Conclusion}

In this paper we present a distributed CS scheme for application in sensor networks to solve the problem of signal estimation. We explore the use of the approximated message passing algorithm in conjunction with gossip algorithms in order to adapt CS to a distributed setting, this 
Fig. 5 a Original signal, b signal overlap for Gossip IHT and, c signal overlap for Gossip AMP
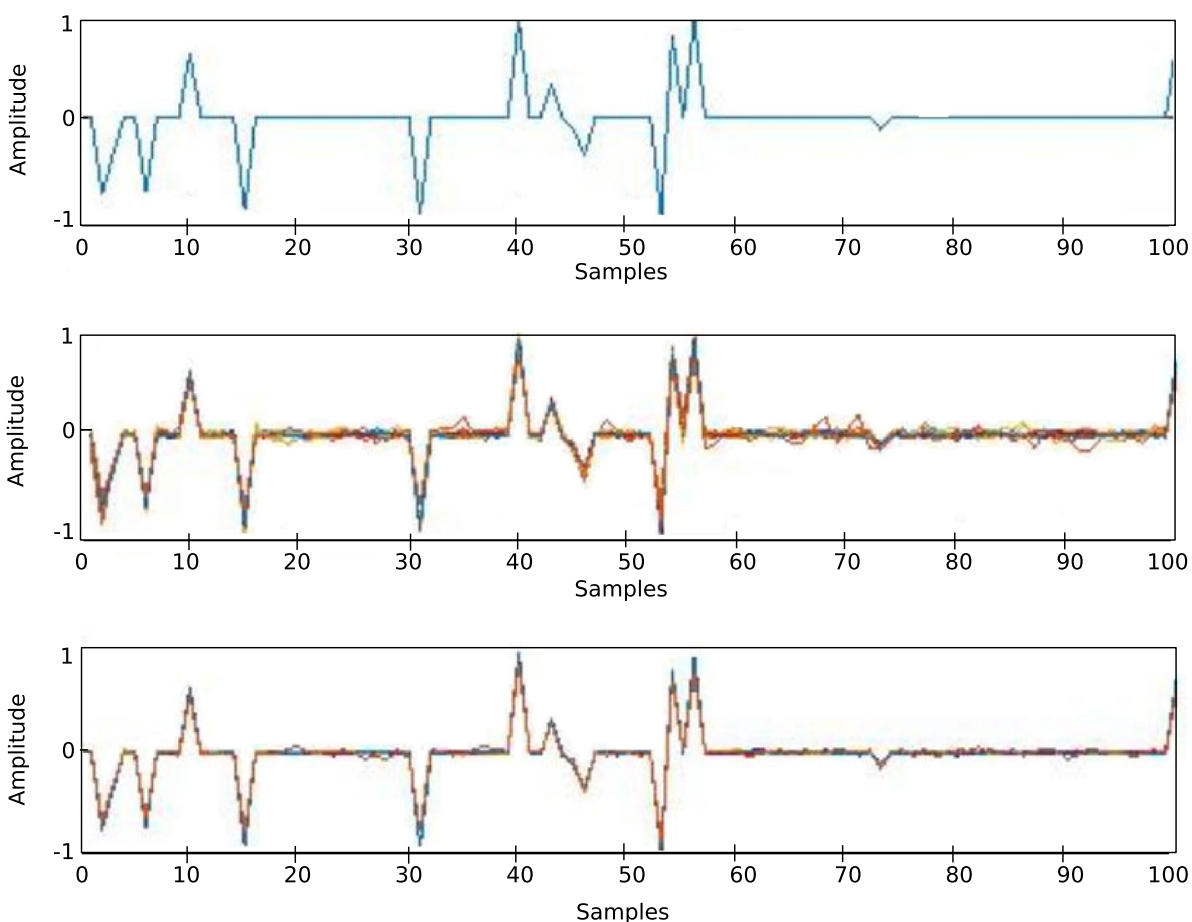

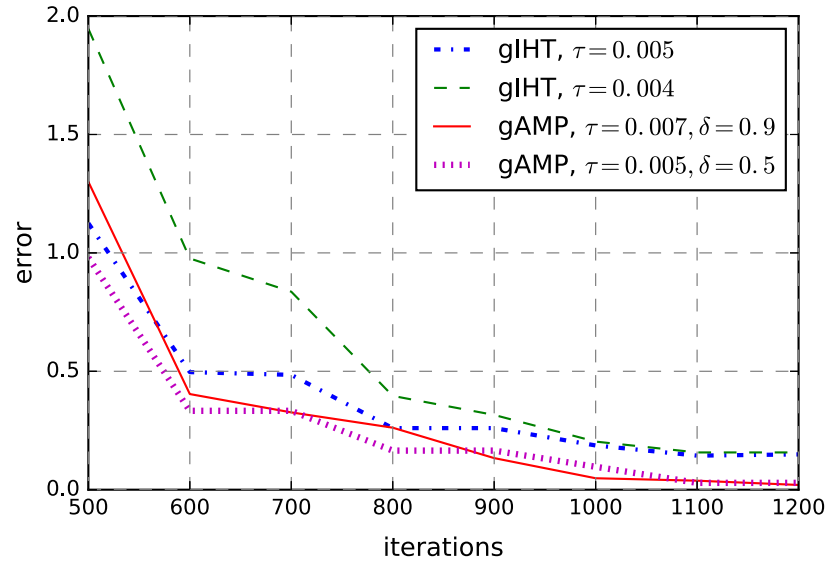

Fig. 6 The graph shows the error of reconstruction of the algorithms for each iteration of the signal. We show the best curves for the gossip IHT (gIHT) and the proposed algorithm, gossip AMP (gAMP)

Table 1 Advantages and disadvantages

\begin{tabular}{ll}
\hline Advantages & Disadvantages \\
\hline $\begin{array}{c}\text { It can work even if each node } \\
\text { has only one neighbor }\end{array}$ & $\begin{array}{c}\text { The parameters of the algorithm } \\
\text { have to be determined } \\
\text { Each step is easy to compute } \\
\text { Only consider the information of } \\
\text { two nodes at each step more } \\
\text { computational time than IHT }\end{array}$ \\
\hline
\end{tabular}

allows a rapid convergence in the reconstruction of signal over a sensor network. The implementation of the proposed algorithm in a wireless sensor network was carried out satisfactorily, we generated a measurement matrix online, which does not have to be stored or transmitted, because it can be generated in each node of the network, using this matrix allows to create a vector of measurements and transmit less number of data in comparison to the size of the original signal.

We carry out experiments using a simulated signals to evaluate a distributed reconstruction using our method, and as comparison, the Gossip IHT was used. We found that our method converges significantly faster than Gossip IHT, and on most cases the error in the reconstruction at each iteration is less in our proposed method than using Gossip IHT. Future research directions include: the use of a regularizing term to reduce possible noise in the signal, to explore diffusion schemes instead of gossip algorithms, a convergence analysis of the algorithm, and the implementation of the algorithm on microprocessors.

\section{References}

1. Aysal, T. C., Yildiz, M. E., Sarwate, A. D., \& Scaglione, A. (2009). Broadcast gossip algorithms for consensus. IEEE Transactions on Signal processing, 57(7), 2748-2761.

2. Baraniuk, R. G. (2007). Compressive sensing [lecture notes]. IEEE Signal Processing Magazine, 24(4), 118-121.

3. Boyd, S., Ghosh, A., Prabhakar, B., \& Shah, D. (2006). Randomized gossip algorithms. IEEE Transactions on Information Theory, 52(6), 2508-2530.

4. Candes, E. J., \& Tao, T. (2006). Near-optimal signal recovery from random projections: Universal encoding strategies? IEEE Transactions on Information Theory, 52(12), 5406-5425. 
5. Cao, M., Spielman, D. A., \& Yeh, E. M. (2006). Accelerated gossip algorithms for distributed computation. In Proceedings of the 44th annual Allerton conference on communication, control, and computation (pp. 952-959). Citeseer.

6. Di Lorenzo, P., \& Scutari, G. (2016). Next: In-network nonconvex optimization. IEEE Transactions on Signal and Information Processing over Networks, 2(2), 120-136.

7. Dimakis, A. G., Kar, S., Moura, J. M., Rabbat, M. G., \& Scaglione, A. (2010). Gossip algorithms for distributed signal processing. Proceedings of the IEEE, 98(11), 1847-1864.

8. Donoho, D. L., Maleki, A., \& Montanari, A. (2009). Messagepassing algorithms for compressed sensing. Proceedings of the National Academy of Sciences, 106(45), 18914-18919.

9. Donoho, D. L., Maleki, A., \& Montanari, A. (2010). Message passing algorithms for compressed sensing: I. Motivation and construction. In 2010 IEEE information theory workshop on information theory (ITW 2010, Cairo) (pp. 1-5). IEEE.

10. Duarte, M. F., Davenport, M. A., Takhar, D., Laska, J. N., Sun, T., Kelly, K. F., et al. (2008). Single-pixel imaging via compressive sampling. IEEE Signal Processing Magazine, 25(2), 83-91.

11. Han, P., Niu, R., Ren, M., \& Eldar, Y. C. (2014). Distributed approximate message passing for sparse signal recovery. In 2014 IEEE global conference on signal and information processing (GlobalSIP) (pp. 497-501). IEEE.

12. Haupt, J., Bajwa, W. U., Rabbat, M., \& Nowak, R. (2008). Compressed sensing for networked data. IEEE Signal Processing Magazine, 25(2), 92-101.

13. Li, S., Da Xu, L., \& Wang, X. (2013). Compressed sensing signal and data acquisition in wireless sensor networks and internet of things. IEEE Transactions on Industrial Informatics, 9(4), 2177-2186.

14. Liu, J., Lian, F., \& Mallick, M. (2016). Distributed compressed sensing based joint detection and tracking for multistatic radar system. Information Sciences, 369, 100-118.

15. Lu, J., Tang, C. Y., Regier, P. R., \& Bow, T. D. (2011). Gossip algorithms for convex consensus optimization over networks. IEEE Transactions on Automatic Control, 56(12), 2917-2923.

16. Lustig, M., Donoho, D. L., Santos, J. M., \& Pauly, J. M. (2008). Compressed sensing MRI. IEEE Signal Processing Magazine, 25(2), 72.

17. Mamaghanian, H., Khaled, N., Atienza, D., \& Vandergheynst, P. (2011). Compressed sensing for real-time energy-efficient ECG compression on wireless body sensor nodes. IEEE Transactions on Biomedical Engineering, 58(9), 2456-2466.

18. Mota, J. F., Xavier, J. M., Aguiar, P. M., \& Puschel, M. (2012). Distributed basis pursuit. IEEE Transactions on Signal Processing, 60(4), 1942-1956

19. Mukhopadhyay, S., \& Chakraborty, M. (2018). Deterministic and randomized diffusion based iterative generalized hard thresholding (DiFIGHT) for distributed sparse signal recovery. arXiv preprint, arXiv:1804.08265.

20. Nedic, A., Olshevsky, A., \& Shi, W. (2017). Achieving geometric convergence for distributed optimization over time-varying graphs. SIAM Journal on Optimization, 27(4), 2597-2633.

21. Nedic, A., Ozdaglar, A., \& Parrilo, P. A. (2010). Constrained consensus and optimization in multi-agent networks. IEEE Transactions on Automatic Control, 55(4), 922-938.

22. Ravazzi, C., Fosson, S., \& Magli, E. (2014). Energy-saving gossip algorithm for compressed sensing in multi-agent systems. In 2014 IEEE international conference on acoustics, speech and signal processing (ICASSP) (pp. 5060-5064). IEEE.

23. Srbinovska, M., Gavrovski, C., Dimcev, V., Krkoleva, A., \& Borozan, V. (2015). Environmental parameters monitoring in precision agriculture using wireless sensor networks. Journal of Cleaner Production, 88, 297-307.

24. Tian, Z., \& Giannakis, G. B. (2007). Compressed sensing for wideband cognitive radios. In 2007 IEEE international conference on acoustics, speech and signal processing-ICASSP'07 (Vol. 4, pp. IV-1357). IEEE.

25. Zaki, A., Venkitaraman, A., Chatterjee, S., \& Rasmussen, L. K. (2018). Greedy sparse learning over network. IEEE Transactions on Signal and Information Processing over Networks, 4(3), 424-435.

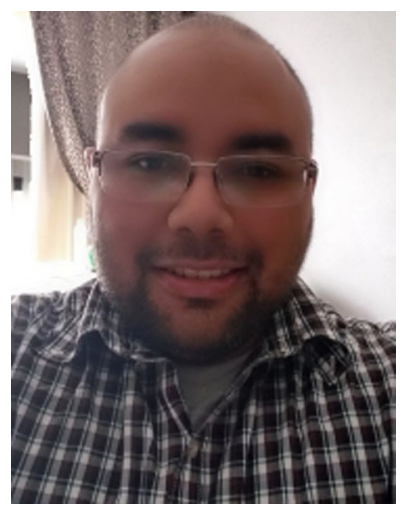

Juan Martinez is a Engineer of Automation at the Servicios Industriales Durol. He holds a master's degree in Electrical Engineering from the Universidad Autonoma de Ciudad Juarez and B.S. in Digital Systems and Comunications from the Universidad Autonoma de Ciudad Juarez. He has published in the Journal ETCM 2016: IEEE Ecuador Technical Chapters Meeting and Congr. Int. Ing. Electron.Mem.Electro 2013.

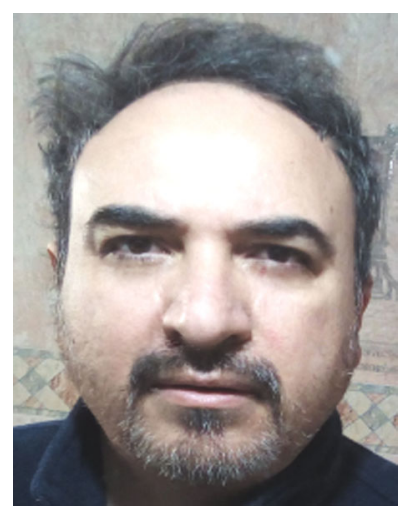

Engineering at the UACJ.

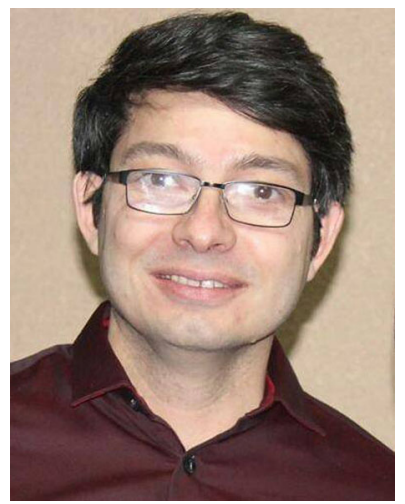

Jose Mejia received the M.Sc. degree in Electrical Engineering and a Ph.D. in Engineering from the Universidad Autonoma de Ciudad Juarez (UACJ), Mexico. $\mathrm{He}$ has also worked for the Consumer Electronics and Automotive Industry as an Electronic Designer. His research interests include sensor networks, medical image reconstruction, and game theory. He is currently a Research Professor in the Department of Electrical and Computation

Boris Mederos has a Ph.D. in Applied Mathematics from the Instituto de Matemática Pura e Aplicada (IMPA), Brazil. He is currently a Full-Time Professor at the Autonomous University of Ciudad Juárez, México. His research interests include medical image processing, machine learning, calculus of variation, optimization and numerical methods. 


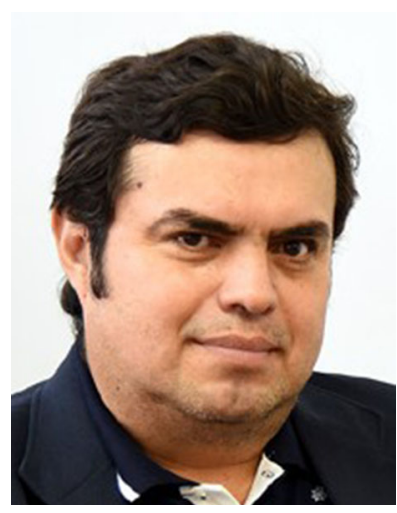

Alberto Ochoa (Bs'94-Eng.Master'00; PhD'04-Postdoctoral Researcher'06 \& Industrial Postdoctoral Research'09). He joined the Juarez City University in 2008 . He participates in the organization of several International Conferences. $\mathrm{He}$ has review of four important Journals from Elsevier: highlighting Applied Soft Computing and Computers in Human Behavior. His research interests include ubiquitous compute, evolutionary computation, natural processing language, social modeling, anthropometrics characterization and Social Data Mining. In May 2016 begin a Sabbatical internship year in Barcelona Supercomputing Center with a project related with simulation of human stampedes and refugee boat sinking improved with Artificial Intelligence. Actually has Level 2 in SNI membership at Conacyt. He has a project with European Union specially with Genoveva Vargas (France) related with Smart Cities.

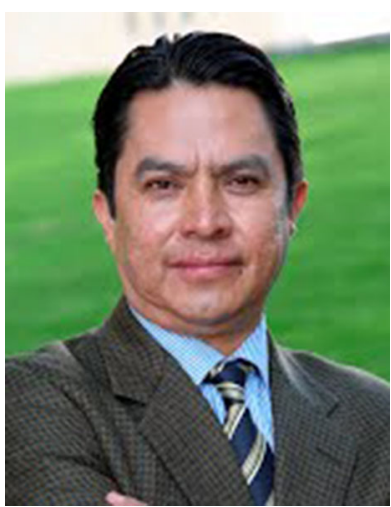

Oliverio Cruz-Mejía is a Professor of Logistics and Transportation at the Universidad Autónoma del Estado de México, México. He holds a Ph.D. in Management Science form the Lancaster University, UK, a master's degree in Manufacturing Engineering from the University of Syracuse, USA and a B.S. in Mechanical Engineering from National Polytechnic Institute of Mexico. $\mathrm{He}$ is board review member of the Journal of Supply Chain Management (JSCMP). He has published in the Journal of Physical
Distribution and Logistics Management (JPDLM), Mathematical Problems in Engineering (MPE), Journal of Ambient Intelligence and Humanized Computing (JAIHC), International Journal of Applied Metaheuristic Computing and Nova Scientia. Oliverio has contributed to book chapters on Simulation for Industry 4.0 (Springer) and Order Picking Performance in Logistics (IGI Global).

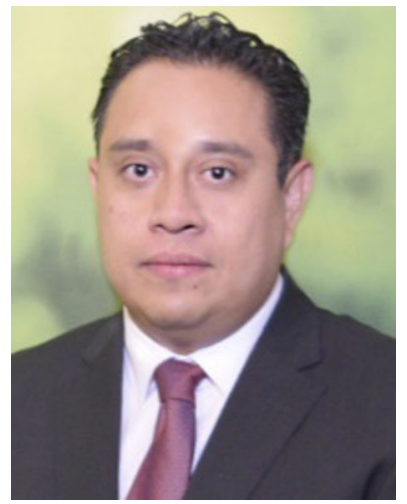

Jose Antonio Marmolejo-Saucedo is a Professor at Panamerican University, Mexico. His research is on operations research, large-scale optimization techniques, computational techniques and analytical methods for planning, operations, and control of electric energy and logistic systems. He is particularly interested in topics related to artificial intelligence, digital twins, the Internet of things and Industry 4.0. $\mathrm{He}$ received his Doctorate in Operations Research (Hons) at National Autonomous University of Mexico. At present, He has the second highest country-wide distinction granted by the Mexican National System of Research Scientists for scienti?c merit (SNI Fellow, Level 2). He is a member of the Network for Decision Support and Intelligent Optimization of Complex and Large Scale Systems and Mexican Society for Operations Research. $\mathrm{He}$ has co-authored research articles in science citation index journals, conference proceedings, presentations, books, and book chapters.

Publisher's Note Springer Nature remains neutral with regard to jurisdictional claims in published maps and institutional affiliations. 